\title{
Routing in Hybrid Mesh Networks of Cognitive and IEEE 802.11 Radios
}

\author{
Marcel William Rocha da Silva and José Ferreira de Rezende \\ Universidade Federal do Rio de Janeiro (UFRJ) \\ Emails: \{marcel,rezende\}@gta.ufrj.br
}

\begin{abstract}
Cognitive radios (CRs) are devices that access spectrum opportunistically taking advantage of unused portions of licensed spectrum. Therefore, depending on the licensed radios activity, cognitive devices may suffer from constant communication disruptions or even lack of opportunities in the access of the spectrum. To solve this problem, we propose a new paradigm of interaction between 802.11 radios and CRs to build hybrid mesh networks. In this architecture every node has $\mathbf{8 0 2 . 1 1}$ interfaces, which offer minimum spectrum access guarantees, and some special nodes possess additional CR interfaces, which may be opportunistically used to create "cognitive shortcut links". These shortcuts improve the hybrid network performance reducing the number of hops in routes and alleviating spectrum sharing in 802.11 channels. In order to create these shortcuts, we propose a shortcut discovering and selecting mechanism that presents a good performance when compared to an optimal case.
\end{abstract}

\section{INTRODUCTION}

Spectrum is becoming an increasingly scarce resource. Aiming at minimizing this problem, the North American Federal Communications Commission (FCC) [1] plans the adoption of new rules that permit an opportunistic access of licensed spectrum by a new class of secondary devices [2].

Cognitive radios (CRs) can be used as secondary devices since they are capable of detecting the operation of licensed radios (also known as primary radios), through sensing and/or consulting databases. Then, they use this information to adapt their own operation parameters to explore the unused portions of spectrum but without causing interference on the primary radios [3], [4]. Thus, CRs can opportunistically use the licensed spectrum, reducing the problem of scarcity due to the increasing demand for spectrum of the existing wireless networks. However, there are still some challenges for the deployment of multi-hop CR networks [5].

One of these challenges is the lack of communication guarantees between cognitive devices operating opportunistically in the licensed spectrum. Because CRs have no priority in accessing the licensed spectrum, the communication between them is very dependent on the activity of primary radios being subject to constant interruptions or even lack of spectrum access opportunities. Therefore, the use of CRs operating exclusively in licensed bands may be inappropriate for most of wireless mesh network (WMN) applications.

To circumvent this problem, this work proposes a new paradigm on the deployment of CRs in WMNs through their integration with 802.11 radios. For the purpose of improving performance, CR interfaces are added to some nodes of a preexistent 802.11 WMN. Through this, the WMN becomes a hybrid network composed by nodes possessing 802.11 and $\mathrm{CR}$ interfaces. An attractive feature of this architecture is that 802.11 radios are used to create an underlying mesh network that provides minimum communication guarantees while the higher communication range of CRs may be opportunistically explored to establish links that will serve as path shortcuts.

Whenever two or more nodes in a multi-hop route in the 802.11 network, henceforth named 802.11 routes, have additional CR interfaces, there may be possible to create links using them to deviate the traffic from the original 802.11 route. Therefore, those nodes with additional CR interfaces, hereafter named cognitive nodes, may establish a cognitive shortcut link. The establishment of this shortcut link has the purpose of reducing the number of hops and alleviating the medium access sharing in the 802.11 channel. Besides, whenever a cognitive shortcut link becomes unavailable, due to licensed devices activity, original 802.11 links may be immediately restored without disrupting communication.

To enable the use of shortcuts, we developed a new mechanism that discovers and selects the best set of shortcuts available on 802.11 routes. It operates on top of each route of the underlying 802.11 mesh network aiming at reducing the number of hops of the original route. A performance evaluation of the shortcut creation mechanism in face of different link weights assignments is presented.

Few works proposed a similar idea of using both 802.11 radios and CRs with the purpose of guaranteeing communication in a WMN [6], [7]. To the best of our knowledge, no other previous work proposed mechanisms to explore the higher communication range of CRs in hybrid scenarios. In [6], the authors presented the idea of using CRs in 802.11 mesh networks to improve performance and enable communication between distant nodes. However, this idea was neither explored nor evaluated.

The work in [7] proposes a mesh network composed by cells where each access point (AP) is able to dynamically decide if its cell should use a channel in the licensed or unlicensed band. This work was the first to present an architecture that is robust to variations in the licensed spectrum availability since the AP can use an unlicensed channel whenever opportunities on the licensed band are unavailable.

The remaining of this work is organized as follows. Section II presents the proposed hybrid mesh network architecture, showing its main advantages and features. In Section III, we present the idea of creating shortcuts in routes of hybrid 


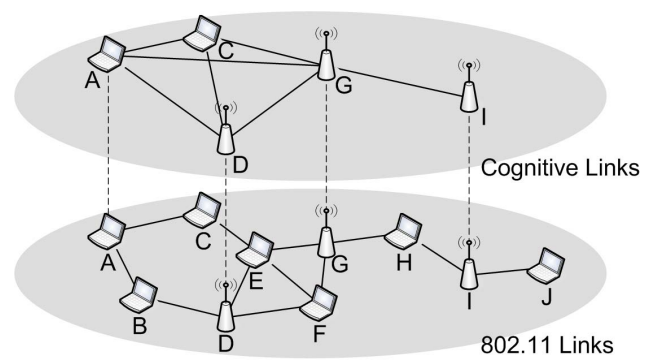

Fig. 1. Hybrid network example

networks and the mechanisms proposed for this purpose. In Section IV, we describe the simulations and discuss the obtained results. Finally, Section V draws some conclusions.

\section{Hybrid Wireless Mesh Networks}

As previously mentioned, the lack of communication guarantees of WMNs composed only by CRs may make their use unfeasible for many applications. CRs are secondary devices that access the licensed spectrum bands in an opportunistic fashion. Whenever a previously unused channel is occupied by a primary device, secondary cognitive devices are forbidden to access that channel with the risk of interfering with the legacy device. Therefore, CRs do not fairly share the spectrum with licensed devices. This unfairness may lead to frequent disruptions on cognitive links or even make them unavailable.

An interesting idea to solve this problem is to consider the use of 802.11 radios along with CRs. 802.11 radios use the unlicensed $2.4 \mathrm{GHz}$ band, where the access is fairly shared among devices through the CSMA/CA protocol.

The wireless mesh hybrid network architecture proposed in this work resembles in some aspects the ideas presented in [6]. It has the objective of creating a WMN with communication guarantees, even when the communication opportunities on the licensed spectrum are scarce or unavailable. In this architecture, all network nodes have one 802.11 interface operating in the $2.4 \mathrm{GHz}$ band, and some of these nodes have an additional cognitive interface operating in the UHF band from $480 \mathrm{MHz}$ to $800 \mathrm{MHz}$.

The nodes of the hybrid network compose an underlying 802.11 network, just as in a conventional 802.11 WMN. Their links are used as immediate backup alternatives whenever the opportunities used by CRs become unavailable, which provides the required communication guarantees. Besides, the higher communication range of CRs, which operates in lower frequencies, can be explored opportunistically to create cognitive shortcut links that reduce the number of hops in the underlying 802.11 routes.

Figure 1 shows an example of a hybrid network. In this scenario, the nodes A, C, D, G and I possess additional cognitive interfaces and may establish new links between them, called hereafter cognitive links. An intuitive way to represent this scenario is to compare it to an overlay network, where the 802.11 is the substrate for the CRs. If node A wishes to communicate with node $\mathbf{J}$, the path with fewer hops in the 802.11 substrate network is composed by nodes A, C, E, G, H,

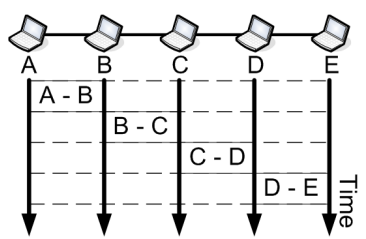

(a) Without shortcut

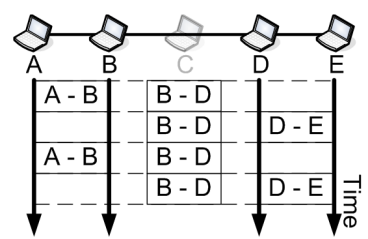

(b) Using shortcut
Fig. 2. Multi-hop communication from node A to node E

I and J. In this case, the route is composed by 6 hops, which would require 6 transmissions for a given frame generated by node A to reach its destination. However, routes with few hops could be created considering the higher distance links between CRs showed in the overlay portion of the figure. In this example, an alternative route could be composed by the cognitive links between A, G and I, and then the link in the 802.11 network between I and J. This way, the communication between $\mathrm{A}$ and $\mathrm{J}$ could be performed with a lesser number of hops through a hybrid route composed of 802.11 and cognitive links.

The next section discusses the advantages of using shortcuts and presents the shortcut discovery and selection mechanisms.

\section{Shortcuts IN Routes}

One of the aspects that adversely affect the throughput of flows in wireless 802.11 mesh networks is the end-to-end number of hops. When numerous transmissions are required to forward a frame from source to destination, intra-flow interference occurs due to the medium access sharing between frames of the same flow, which also increases latency.

Figure 2(a) shows an example of multiple hop communication between nodes $\mathrm{A}$ and $\mathrm{E}$. Each time window shows the possible concurrent frame transmissions. Considering that the carrier sense detection range is twice the communication range, while node A transmits a frame to node $\mathrm{B}$, stations $\mathrm{C}$ and $\mathrm{D}$ are not allowed to transmit since they are within carrier sense range of nodes A and B. Thus, only one of the links in the route may be used to transmit frames per time window. This reduces drastically the end-to-end throughput of the flow.

Considering now that stations $\mathrm{B}$ and $\mathrm{D}$ possess additional $\mathrm{CR}$ interfaces whose communication range is twice that of 802.11 radios, one can construct a cognitive shortcut link between $\mathrm{B}$ and $\mathrm{D}$ employing an unused channel of licensed band (Figure 2(b)). At this new scenario, nodes A and B (or nodes B and D) can transmit simultaneously. This occurs because stations $\mathrm{B}$ and $\mathrm{D}$ possess two interfaces and may receive and transmit frames in the licensed band at the same time they are receiving or transmitting frames using the 802.11 interface in the unlicensed band. Thus, by deviating frames from the flow between A and E through this shortcut link, the communication is performed with fewer hops, increasing the throughput and reducing the end-to-end delay.

In addition, the creation of cognitive shortcuts in the hybrid mesh network has the beneficial effect of reducing the number of transmissions in the unlicensed band. Therefore, the use of shortcuts also has the favorable effect of reducing the 802.11 
network load, which improves performance of underlying 802.11 communications.

\section{A. Shortcut Creation Mechanism}

The shortcut creation mechanism works on top of previously created 802.11 routes. Whenever a cognitive node detects that it is forwarding frames of a flow between a sourcedestination pair, it starts sending probes in the direction of both source and destination. Those control probes contain the id of the cognitive node and its available spectrum access opportunities in the licensed band. After receiving these probes, the cognitive nodes in the route have discovered each other and their respective spectrum access opportunities. Using this information along with a specialized MAC protocol, such as the ones presented in [8], [9], the cognitive nodes may coordinate themselves by scanning opportunities and sending other control messages to determine which are the possible shortcut links that can be created in that specific route.

After that, a new probing phase starts, in which the cognitive nodes periodically send beacons to both the source and destination. These probes carry the information regarding the possible shortcut links that can be created using that cognitive node. Thus, at the end of this phase, all cognitive nodes of that route know all the possible shortcuts that can be created. However, only a subset of them can be used simultaneously.

Considering that the routing protocol of the 802.11 WMN provides complete route information, the cognitive node closest to the source is able to select the best subset of shortcuts to be used. For this purpose, it builds a partial view of the topology graph, containing the links of the 802.11 route and all possible discovered shortcuts. All links in this partial graph are assigned equal weights and used as input to a SPF (Shortest Path First) algorithm. This algorithm provides the shortest hybrid route in that graph by using the best set of shortcuts available. It is important to notice that other performance metrics can be optimized by using other link weight metrics in the partial graph. However, the equal link weights are well suited to the purpose of this work, which is to evaluate the reduction in number of hops by using cognitive shortcut links.

\section{B. Integration with Routing Protocol of the 802.11 WMN}

The entire process of shortcut creation is closely related to the routing protocol of the underlying 802.11 WMN because the proposed mechanism operates on top of the routes discovered in the 802.11 network. Therefore, the availability of shortcuts in 802.11 routes depends on the routing protocol of the 802.11 WMN. To understand this influence, we evaluate different ways of creating 802.11 routes considering a proactive routing protocol, such as OLSR [10].

In OLSR, a SPF algorithm is used to discover routes where the sum of link weights is minimized. Hence, the route discovery process is guided by the link weight metric. A usual metric is to assign unit weights to all links. However, this is not expected to be a good approach in our hybrid network scenario because this metric does not take into account information about the cognitive nodes. In the following, we propose two link weight metrics that can be used by the routing protocol of the underlying 802.11 WMN with the aim of increasing the probability that shortcuts exist.

1) NUMCR: Link weights are determined by the parameter $C R_{x}$ in equation 1 , which is a binary variable that assumes value 1 if node $x$ has an additional cognitive interface, and 0 otherwise. The goal of this metric is to favor the composition of routes that have preferentially higher number of CRs, increasing the probability of shortcut creation. The constant $N$ is used in equation 1 to weight the influence of parameters $C R_{i}$ and $C R_{j}$ in link weights.

$$
\text { Weight }_{N U M C R}(i, j)=\frac{1}{N \times\left(C R_{i}+C R_{j}\right)+1}
$$

2) PERCENTOP: Besides considering whether the nodes composing the link have cognitive interfaces, the percentage of spectrum access opportunities of each node is taken into account. This percentage is determined by the amount of opportunities available to the node divided by the total amount of opportunities in the licensed band. In equation 2, the weight of the link between nodes $i$ and $j$ is given by their percentage of opportunities available, $O P_{i}$ and $O P_{j}$, and the constant $N$ has the same purpose as in equation 1 . Therefore, this metric favors the creation of routes with cognitive nodes that have high percentage of spectrum access opportunities available.

$$
\text { Weight }_{P E R C E N T O P}(i, j)=\frac{1}{N \times\left(O P_{i}+O P_{j}\right)+1}
$$

\section{Straightforward Hybrid Routes Discovery}

Another way to create hybrid routes is to modify the routing protocol of the 802.11 WMN to consider the cognitive links in the route discovery process. The SPF algorithm may use a full joint network map as input, composed by 802.11 and cognitive links with equal weights. This modified routing protocol can discover the shortest hybrid routes in the network.

It is clear that this approach is straightforward to determine short hybrid routes. However, the discovered hybrid routes are not attached to underlying 802.11 routes. This way, in case of cognitive links disruption in the hybrid route, it would not be possible to instantly fallback into an 802.11 backup route. If the cognitive links availability changes fast in time due to the licensed devices activity, the hybrid routes created by the modified routing protocol should have short lifetime. Moreover, the lack of an 802.11 backup route obliges the routing protocol to perform another route discovery, during which frames in transit may be lost. This problem reduces the communication quality, which would suffer from constant disruptions, and also overloads the routing protocol due to the constant route recovery process.

A way to circumvent this issue is to determine backup routes for each cognitive link in the hybrid route. However, in some specific scenarios it is not possible to determine backup routes for every cognitive link in a hybrid route without creating routing loops. Especially in hybrid routes that pass through cognitive nodes that have few links at the 802.11 network. If such cognitive node participates in two links of the hybrid 
route, the backup routes of both links may need to pass through the same 802.11 nodes, creating a routing loop.

\section{Simulations}

A simulator that generates a hybrid network scenario was developed to evaluate the shortcut creation mechanism. It randomly generates network topologies composed by 802.11 and cognitive links, and implements a SPF algorithm. In the experiments, the SPF is executed taking as input the 802.11 links to determine all possible routes, as in a proactive routing protocol. We performed experiments by assigning equal link weights to all links (UNIT) and the two proposed link weight metrics (NUMCR and PERCENTOP). After that, the shortcut creation mechanism is executed on top of each route.

We also simulated the straightforward hybrid routes discovery using the complete network topology as input to the SPF algorithm. As stated in the Section III-C, this simulation provides the shortest hybrid routes that will serve as a benchmark to our proposal (OPTIMAL). To determine the backup routes for these hybrid routes, we implemented a simple greedy algorithm that tries to determine backup routes for each cognitive link used in the hybrid routes. Before creating a backup route to each cognitive link and in order to avoid loops, it removes from the network topology all predecessor hops of the hybrid route and all nodes from the backup routes already discovered. By this reason, in some cases it is not possible to determine backup routes for some cognitive links.

\section{A. Simulation Environment and Methodology}

For the simulations, we generated 30 scenarios comprised by a square area of $1000 \mathrm{~m} \times 1000 \mathrm{~m}$ and 200 nodes randomly disposed with the following restrictions imposed: (i) each node deployed must be in communication range of any already deployed node; (ii) the neighborhood degree does not exceeds a maximum degree of 6; (iii) a minimum distance of $20 \mathrm{~m}$ between nodes was respected. Hence, the generated scenarios were fully connected and sparse, resembling typical WMNs, such as community networks of a large city. The results presented are mean values of the 30 scenarios with confidence interval bounds at a confidence level of $95 \%$.

Each node possesses an 802.11 radio interface with communication range of 80 meters. Additionally, some randomly selected nodes possess a CR interface with communication range of 320 meters, i.e. 4 times the 802.11 range. Considering 802.11 radios operating in $2,4 \mathrm{GHz}$ spectrum and a free space propagation model, this would represent a $\mathrm{CR}$ operating in the $600 \mathrm{MHz}$ spectrum band.

The licensed band considered in the simulations was composed of 10 channels. For each channel, two communicating pairs of primary radios with 320 meters communication range were randomly placed in the scenarios. A channel is available to a CR if it is not in the communication range of any primary radio using that channel.

\section{B. Results}

Figure 3 shows the average number of hops of the routes between all possible source-destination pairs in the network as

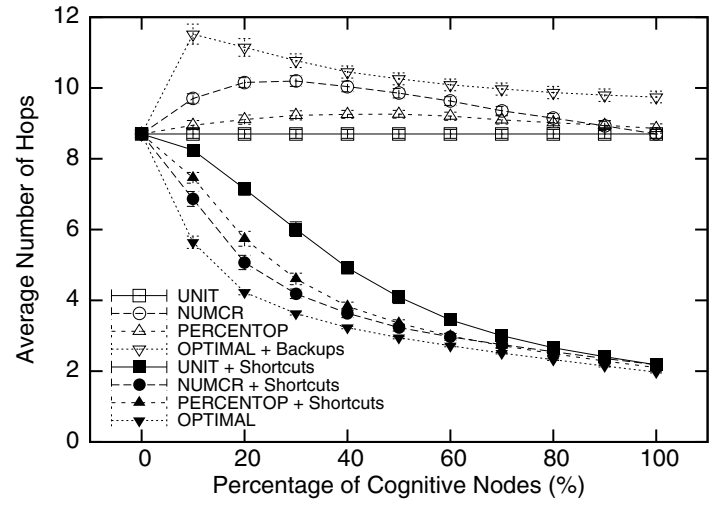

Fig. 3. Average number of hops as a function of cognitive nodes percentage

function of the percentage of CRs. This is an important metric since the objective of the shortcut creation mechanism is to reduce the number of hops in the 802.11 routes. Each curve in the graph show the results obtained by using a different weight metric, before and after the execution of the shortcut creation mechanism. At these experiments, NUMCR and PERCENTOP have used $N=2$. For the straightforward hybrid routes discovery, there are two curves named as OPTIMAL, before and after the greedy backup discovery algorithms' execution.

As expected, the average number of hops of the routes with shortcuts is inversely proportional to the percentage of cognitive nodes since the higher communication range of CRs allows the connectivity of distant nodes with fewer hops. An interesting observation is that the shortcut creation mechanism creates hybrid routes with a number of hops close to the optimal. One may also notice that a great part of those gains occurs due to the specialized routing metrics, NUMCR or PERCENTOP. Those results confirm that using cognitive nodes information in the process of 802.11 underlying routes discovery is favorable to the shortcut selection algorithm.

Comparing NUMCR and PERCENTOP metrics, one can see that NUMCR is able to provide better opportunities for the shortcut creation mechanism to produce hybrid routes close to the optimal number of hops than PERCENTOP. However, one can also see that NUMCR increases the average number of hops of the 802.11 backup routes. In scenarios where the cognitive links have highly variable availability, PERCENTOP metric is expected to perform better than NUMCR because most of the time the packets will have to be forwarded through the 802.11 backup routes which are shorter using PERCENTOP. The same problem occurs when using the OPTIMAL approach described in Section III-C. The paths composed by the 802.11 backup routes created by the greedy algorithm are longer than the 802.11 routes created by the SPF algorithm with any routing metric. Therefore, besides the shortcoming of being unable to have backups for all cognitive links, this approach would present worse performance in scenarios of dynamic cognitive links availability.

Figure 4 presents the distribution of the number of hops in routes from the scenarios where $30 \%$ of the nodes have cognitive interfaces. One can see that before the shortcut creation 


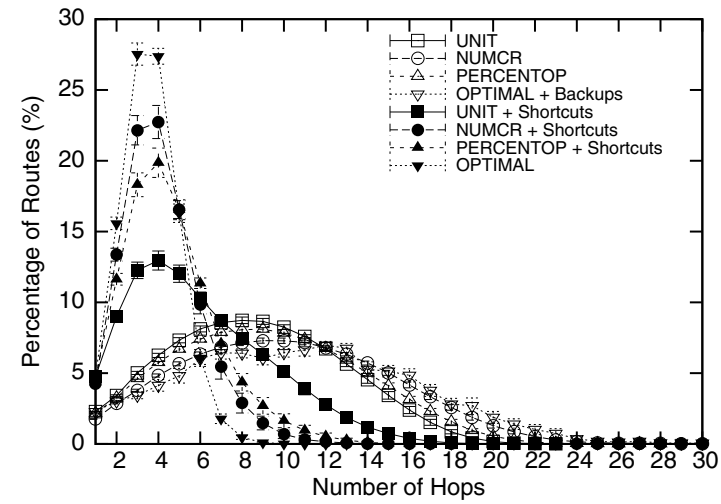

Fig. 4. Percentage of routes as a function of the number of hops with $30 \%$ of cognitive nodes

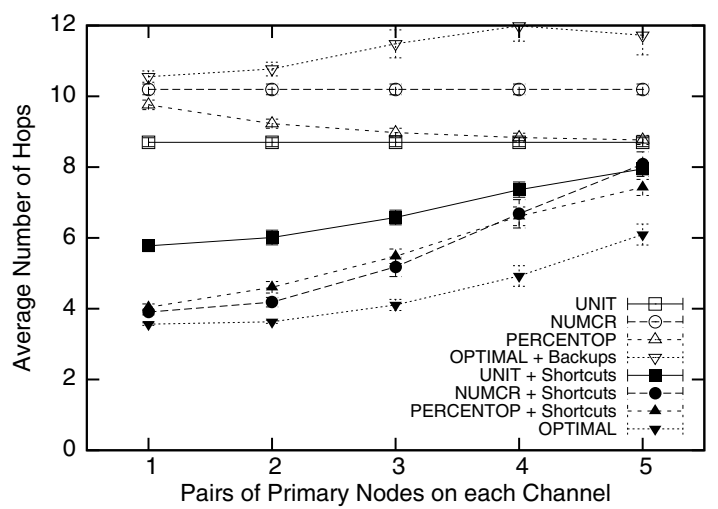

Fig. 5. Average number of hops as a function of cognitive nodes percentage with $30 \%$ of cognitive nodes

almost $100 \%$ of the 802.11 routes created with NUMCR have up to 22 hops. However, using shortcuts the maximum number of hops is reduced to 10 . This result shows that the reduction on the average number of hops generated by the shortcuts was obtained over the longer routes, which are the ones that present worse performance for multi-hop communications.

Figure 5 shows the average size of the routes as a function of the number of primary pairs, which directly influences the availability of spectrum access opportunities to CRs. In scenarios with few opportunities, PERCENTOP metric creates shorter 802.11 routes than NUMCR. Moreover, using either NUMCR or PERCENTOP the hybrid routes created by the shortcut creation mechanism have similar performance. It happens because, different from NUMCR, PERCENTOP only favors the creation of 802.11 routes that pass through cognitive nodes with high availability of spectrum opportunities, which are rare in such scenarios. Therefore, PERCENTOP do not unnecessarily increases the number of hops of 802.11 routes. Other important result is that the sizes of the hybrid routes created by the shortcut creation mechanism are less efficient in comparison to OPTIMAL approach. However, the better performance of OPTIMAL has the high price of increasing the size of its 802.11 backup routes, which in dynamic scenarios would provide worse performance.

We also performed simulations to evaluate the influence of $N$ parameter in NUMCR and PERCENTOP, with values ranging from 1 to 4 . Using both metrics, an increase in the $N$ value causes the 802.11 routes to be longer and the respective hybrid routes to be closer to the optimal result. However, for all simulated $N$ values, the 802.11 routes created using PERCENTOP have less hops than using NUMCR, and the hybrid routes of PERCENTOP have a number of hops close to NUMCR. This behavior occurs because PERCENTOP creates 802.11 routes that traverse nodes that have high availability of opportunities, which favors shortcuts creation without a large increase in 802.11 route sizes.

\section{CONCLUSions}

The application of CRs in WMNs is limited by the lack of communication guarantees due to its opportunistic behavior when accessing licensed bands. In this work, we proposed a new hybrid network architecture composed by CRs and 802.11 radios. The 802.11 radios compose the underlying network, providing minimum spectrum access guarantees while the higher communication range of CRs is used to create shortcuts in the underlying 802.11 routes, composing hybrid routes.

Since the discovery and selection of the best shortcuts to be used in each route is not straightforward, we proposed a shortcut creation mechanism that aims at reducing the number of hops in the routes. Through simulations, we evaluated its efficiency using different configuration of the routing protocol of the underlying 802.11 WMN. We also compared its performance to a benchmark routing protocol which determines optimum hybrid routes. Simulation results show that the proposed mechanism presents a performance close to the optimum case in most scenarios.

\section{REFERENCES}

[1] FCC, "Federal Communication Commission (FCC) Home Page," 2009, http://www.fcc.gov - last access in 17/11/2009.

[2] — "Unlicensed Operation in the TV Broadcast Bands: Additional Spectrum for Unlicensed Devices Below $900 \mathrm{MHz}$ and in the $3 \mathrm{GHz}$ Band," Nov. 2008, FCC 08-260.

[3] I. F. Akyildiz, W.-Y. Lee, M. C. Vuran, and S. Mohanty, "NeXt Generation/Dynamic Spectrum Access/Cognitive Radio Wireless Networks: A Survey," Computer Networks, vol. 50, no. 13, pp. 2127 - 2159, 2006.

[4] Q. Zhao and B. M. Sadler, "A Survey of Dynamic Spectrum Access: Signal Processing, Networking, and Regulatory Policy," IEEE Signal Processing Magazine, pp. 79-89, May 2007.

[5] I. F. Akyildiz, W.-Y. Lee, and K. R. Chowdhury, "CRAHNs: Cognitive radio ad hoc networks," Ad Hoc Networks, vol. 7, no. 5, pp. 810-836, 2009.

[6] J. A. Stine, "Spectrum Management: The Killer Application of Ad Hoc and Mesh Networking," DySPAN '05: IEEE International Symposium on New Frontiers in Dynamic Spectrum Access Networks, Nov. 2005.

[7] K. R. Chowdhury and I. F. Akyildiz, "Cognitive Wireless Mesh Networks with Dynamic Spectrum Access," IEEE Journal on Selected Areas in Communications, vol. 26, no. 1, pp. 168-181, Jan. 2008.

[8] C. Cordeiro and K. Challapali, "C-MAC: A Cognitive MAC Protocol for Multi-Channel Wireless Networks," in DySPAN '07: IEEE International Symposium on New Frontiers in Dynamic Spectrum Access Networks, Apr. 2007.

[9] Y. R. Kondareddy and P. Agrawal, "Synchronized MAC Protocol For Multi-Hop Cognitive Radio Networks," in ICC '08: IEEE International Conference on Communications, May 2008.

[10] T. Clausen, P. Jacquet, C. Adjih, A. Laouiti, P. Minet, P. Muhlethaler, A. Qayyum, and L.Viennot, "Optimized Link State Routing Protocol (OLSR)," RFC 3626, October 2003, network Working Group. [Online]. Available: http://ietf.org/rfc/rfc3626.txt 